\title{
THEORETICAL FRAMEWORK OF AGENT ASSISTED TREATMENT FOR ASD (AUTISM SPECTRUM DISORDER)
}

\author{
DANIYAL HUSSAIN BUTT ${ }^{1}$, WASIM AHMAD KHAN ${ }^{2}$ \\ ${ }^{1}$ Department of Computer Sciences, National College of Business Administration \& Economics \\ Lahore, Pakistan \\ ${ }^{2}$ Department of Computer Sciences, National College of Business Administration \& Economics \\ Lahore, Pakistan \\ Email: \{ danialhussain1994 ${ }^{1}$, wasimahmad.ucit ${ }^{2}$ \} @ gmail.com \} \\ ABSTRACT. kids with Autism Spectrum problems (ASD) have conversation deficits \\ and problems with social interaction. A lack of social conduct can bog down healing \\ interventions and may lessen the potential to examine social talents. Robots had been \\ shown to initiate proactive social conduct in youngsters with ASD. improvement of \\ robot systems able to appearing as catalysts for social conduct in the context of ASD \\ remedy is the need of contemporary era. A methodology and theoretical framework is \\ being supplied here in this paper for a robotic system that can not only compare the \\ effects and stage of ASD however can assist the victim consequently. \\ Keywords: ASD; Robotic System; Victim; Social Conduct.
}

1. Introduction. Autism spectrum disorder (ASD) is a neuro and evolving disease that starts off evolved early in formative years and lasts all over an individual's life. It impacts how somebody acts and interrelates with others, learns and share knowledge. It includes what was once called Asperger syndrome and pervasive developmental problems [1]. it is called a "spectrum" disease due to the fact human beings with ASD will have a number of symptoms. humans with ASD might have issues speaking with you, or they won't appearance you in the attention while you talk to them. they will additionally have limited pastimes and repetitive behaviors. they will spend a whole lot of time placing matters so as, or they will say the equal sentence again and again. [2] they will often seem to be of their "very own global. "The causes of ASD aren't recognized. research indicates that each genes and surroundings play crucial roles. there's currently no one standard remedy for ASD. there are numerous methods to boom infant's capacity to develop and examine new competencies. beginning them early can lead to better outcomes. remedies encompass conduct and conversation therapies, skills schooling, and drugs to control signs. Symptoms and Signs of ASD:

$>$ Speaking: Symptoms and signs of ASD can be found in speaking as the kids with ASD will have deferred speech growth, repetition of phrases and words will be a lot more than usual, flat or monotonous speech, will prefer to use one word [3].

$>$ Responding to others: Responding to people other than they know is another factor that they will not response to their name, rejects cuddles from parent or care taker and will react negatively sometimes when asked to do something [4].

$>$ Interacting with others: While interacting with others kids with ASD does not know of other's personal space, they have less curiosity in interrelating with other people - as well as kids of a same age, do not enjoy in circumstances that most kids of their age like, they prefer to play alone, these kids rarely use expressions or gestures, they avoid eye contact as much as they can.

> Behavior: ASD patients have tedious developments, for example, fluttering their hands, shaking forward and backward, or flicking their fingers, play with toys in a redundant and bland path, for example, arranging hinders arranged by size or shading, as opposed to utilizing them to construct something, likes to have a recognizable routine and getting exceptionally resentful if there are 
changes to this schedule, have a solid like or aversion of specific sustenance in light of the surface or shade of the nourishment as much as the taste, they have surprising tactile interests - for instance, youngsters with ASD may sniff toys, protests or individuals improperly Individuals with ASD regularly have side effects or parts of different conditions, for instance, A learning inability, Attention deficiency hyperactivity issue (ADHD), Tourette's disorder or other tic issue, Epilepsy, Dyspraxia, Obsessive enthusiastic issue (OCD), Generalized nervousness issue, Depression, Bipolar issue, Sleep issues, Sensory challenges [6].

$>$ Eye-Gaze: Works by having lights and cameras that are continually sending and accepting data. The camera grabs light reflections from your understudies and interprets the development of your eyes into mouse cursor developments. It takes just seconds to finish a one-time adjustment [7].

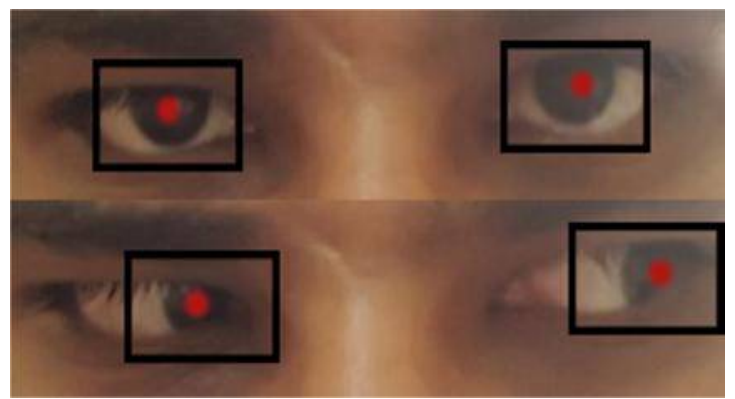

Figure. 1: Eye Gaze Detecetion Using Eye Tribe.

$>$ Facial Expression Detection: Apparently reductive, numerous outward appearance discovery apparatuses protuberance human feeling into 7 primary classes: Happiness, Bitterness, Outrage, Dread, Amazement, Hatred, and Sicken. With facial feeling identification, calculations distinguish faces inside a photograph or video, and sense smaller scale appearances by breaking down the connection between focuses on the face, in view of curated databases gathered in scholarly conditions [8] [9].

$>$ Causes of Extreme Introvertedness(ASD): Specialists are as yet dubious about every one of the reasons for a mental imbalance. Probably, there are different causes - instead of only one. It gives off an impression of being that various diverse conditions including natural, biologic, and hereditary components - set the phase for a mental imbalance and make a youngster more prone to have the turmoil.

There is motivation to trust that qualities assume a noteworthy part in the development of ASD. It has been discovered that indistinguishable twins will probably both be influenced than twins who are intimate (not hereditarily indistinguishable). In a family with one mentally unbalanced youngster, the shot of having another tyke with extreme introvertedness is around 5 percent - or one of every 20 - significantly higher than in the typical populace.

Now and again, guardians or different relatives of a mentally unbalanced tyke have gentle social disabilities, (for example, dreary practices and social or correspondence issues) that look particularly like extreme introvertedness. Research additionally has discovered that some enthusiastic issue, (for example, hyper discouragement) happen all the more regularly in groups of a kid with extreme introvertedness. No less than one gathering of scientists has discovered a connection between a strange quality and a mental imbalance. The quality might be only one of three to at least five qualities that collaborate somehow to cause the condition. Researchers speculate that a defective quality or qualities may make a man more inclined to create a mental imbalance when there are additionally different variables present, for example, a substance unevenness, infections or chemicals, or an absence of oxygen during childbirth. In a couple of cases, mentally unbalanced conduct is caused by:

- Rubella (German measles) in the pregnant mother.

- Tuberous sclerosis (an uncommon hereditary issue that makes considerate tumors develop in the mind and also in other essential organs).

- Delicate $X$ disorder (the most widely recognized acquired type of scholarly inability). 
- Encephalitis (cerebrum irritation).

- Untreated phenylketonuria (PKU) - when the body does not have a catalyst required for ordinary digestion.

In the previous quite a while, there has been enthusiasm for a hypothesis that recommended a connection amongst a mental imbalance and the utilization of thimerosal, a mercury-based additive utilized as a part of the measles-mumps-rubella (MMR) immunization. In spite of the fact that mercury is never again found in youth immunizations in the Assembled States, a few guardians still have worries about inoculations.

In any case, some well-done, extensive scale considers have now been played out that have neglected to demonstrate a connection amongst thimerosal and a mental imbalance. A board from the Organization of Solution is presently looking at these investigations. The reports incorporate a substantial Danish investigation that reasoned that there was no causal connection between youth immunization utilizing thimerosal-containing antibodies and the improvement of an extreme introvertedness range issue and a U.S. think about taking a gander at presentation to mercury, lead, and other substantial metals. Both the American Foundation of Pediatrics and the Communities for Infection Control and Anticipation asert that there is no connection amongst a mental imbalance and the MMR antibody or some other immunization.

Other potential reasons for extreme introvertedness are ecological poisons, including pesticides and substantial metals, for example, mercury. Substantial metals are surely more normally experienced in the earth now than they were before. It might be that individuals with a mental imbalance or those at higher hazard for creating it are touchier than others to these poisons.

$>$ H. Kinect (For body movement detection): The Kinect contains three fundamental sorts that work out to distinguish your movement and make your physical picture on the screen: a RGB shading VGA camcorder, a profundity sensor, and a multi-exhibit mouthpiece. The camera distinguishes the red, green, and blue shading parts and in addition body-sort and facial highlights. It has a pixel determination of 640x480 and an edge rate of $30 \mathrm{fps}$. This aides in facial acknowledgment and body acknowledgment. The profundity sensor contains a monochrome CMOS sensor and infrared projector that assistance make the 3D symbolism all through the room. It likewise measures the separation of each purpose of the player's body by transmitting imperceptible close infrared light and estimating its "season of flight" after it reflects off the articles. The amplifier is really a variety of four mouthpieces that can detach the voices of the player from other foundation clamors enabling players to utilize their voices as an additional control highlight. These segments meet up to identify and track 48 distinct focuses on every person's body and rehashes 30 times each second. Putting both equipment and programming together enable the Kinect to create 3D pictures and perceive individuals inside its field of vision. It can investigate the individual before it and experience numerous "channels" to attempt and figure out which kind of body structure matches with the right sort customized in its framework. Information is always being exchanged forward and backward between the Kinect and the articles in its field of vision. Since the Kinect has an infrared projector, infrared camera and shading camera, it's an extraordinary imaging apparatus, notwithstanding for robots. With a specific end goal to upgrade the range and self-sufficient nature of robots, they should have the capacity to see the earth around them. One way they do this is through synchronous limitation and mapping, or Hammer. Generally, these sorts of sensors are either costly and lumbering or shabby and untrustworthy. Laser exhibits are costly and substantial and can just guide in two measurements. Stereo cameras are light and can outline 3D, however require enormous registering power. Ken Conley of Willow Carport would now be able to offer his Kinect-prepared TurtleBot for $\$ 500$. An immense reserve funds from the past non-Kinect variant that cost over $\$ 250,000$.

\section{Related Work.}

$>$ Biped humanoid agent to imitate human dances: The point of this exploration was to build up a biped humanoid robot that can examine a human move execution and mimic it. To pick up this objective, they proposed an endeavor rendition of lower outline development, which comprised of venture natives (what to do) and capacity parameters (how to do it). based absolutely in this model, a progression of wander natives and their expertise parameters have been recognized from human movement, and robot movement is recovered from the identified outcome underneath requirements of an automated. This adaptation could create human-like lessening outline development comprising 
of various midriff movements notwithstanding different venturing movements of the legs [10]. Created movements can be performed steadily on a real robot upheld by method for its own legs. They utilized advanced robot equipment HRP-2, which had prevalent capacities in body weight, actuators, and DOF of the abdomen. by methods for the utilization of the proposed approach and HRP-2, we have understood a move general execution of jap individuals move by means of the mechanical, that is synchronized with an execution of a human fabulous ace on the equivalent level.

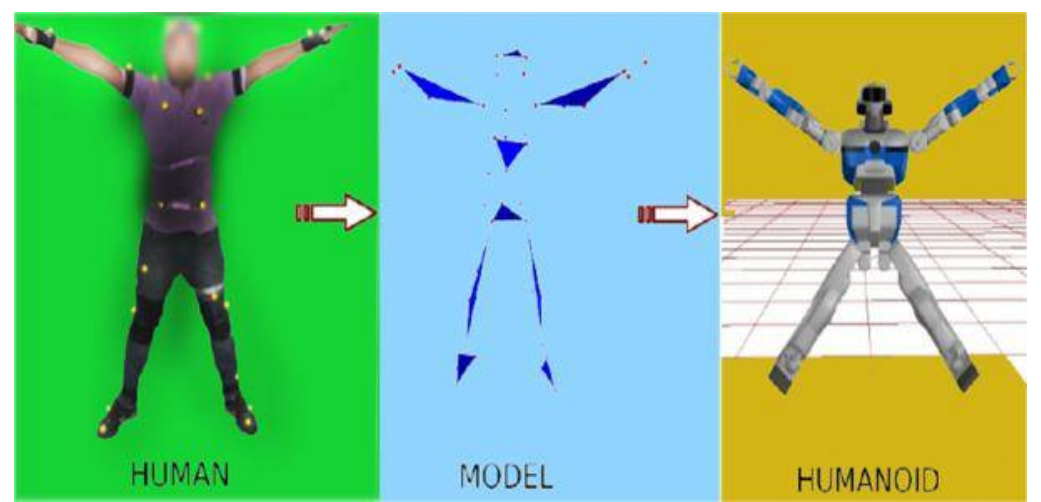

Figure. 2: Body Motion Capturing using Kinect 2.0.

Kinect in autonomous Robots: The Microsoft X-Box Kinect Sensor is a progressive new profundity camera that is utilized as a part of the gaming business to catch movements of individuals and players proficiently utilizing the innovation of a RGB camera and infrared camera to separate profundity. In the Microsoft X-Box, Kinect was utilized to detect 3D impression of human's movements. It can likewise be utilized for automated applications, exactly for indoor route through the procedure of figuring out. Certain product bundles were made accessible and are open source from "LibFreenect" for Linux machines, Microsoft's Kinect SDK utilizing the Kinect namespace on Visual Studio 2010 Express (C++, C\# or Visual Fundamental), and Google's discharged "Automated Working Framework (ROS)". Keeping in mind the end goal to guarantee that this sensor is equipped for going up against such an undertaking, we should have the capacity to explore altogether all factors that add to this and in the meantime, the capacity should be there to comprehend its confinements to be connected and coordinated legitimately with specific sorts of robots for achieving our motivation of accomplishing effective indoor route utilizing appropriate calculations. The outcomes from testing the Kinect sensor on a self-sufficient ground vehicle are being used to further achieve the autonomous nature of robots.

$>$ Vision implementation of head pose and gaze direction measurement: To build wise human interfaces, it's far imperative for a framework to perceive a client's motivation and purpose of consideration. for the reason that development of a man's head stance and look course are profoundly related with his/her objective and intrigue, discovery of such records might be used to build normal and instinctive interfaces. Real time stereo face following and look location gadget to degree head stance and look course all the while is introduced. The fundamental factor of machine was utilizing continuous stereo vision together with a simple arrangement of tenets that is proper for constant handling. since the 3D directions of the abilities on a face might be specifically estimated in machine, It could generously disentangle the arrangement of principles for three-D display fitting to accomplish the full 3-D posture of the best contrasted and conventional structures that utilization monocular digicam. thusly, A non-touch, aloof, ongoing, solid, precise and minimal measurement device for head stance and look way was executed. 


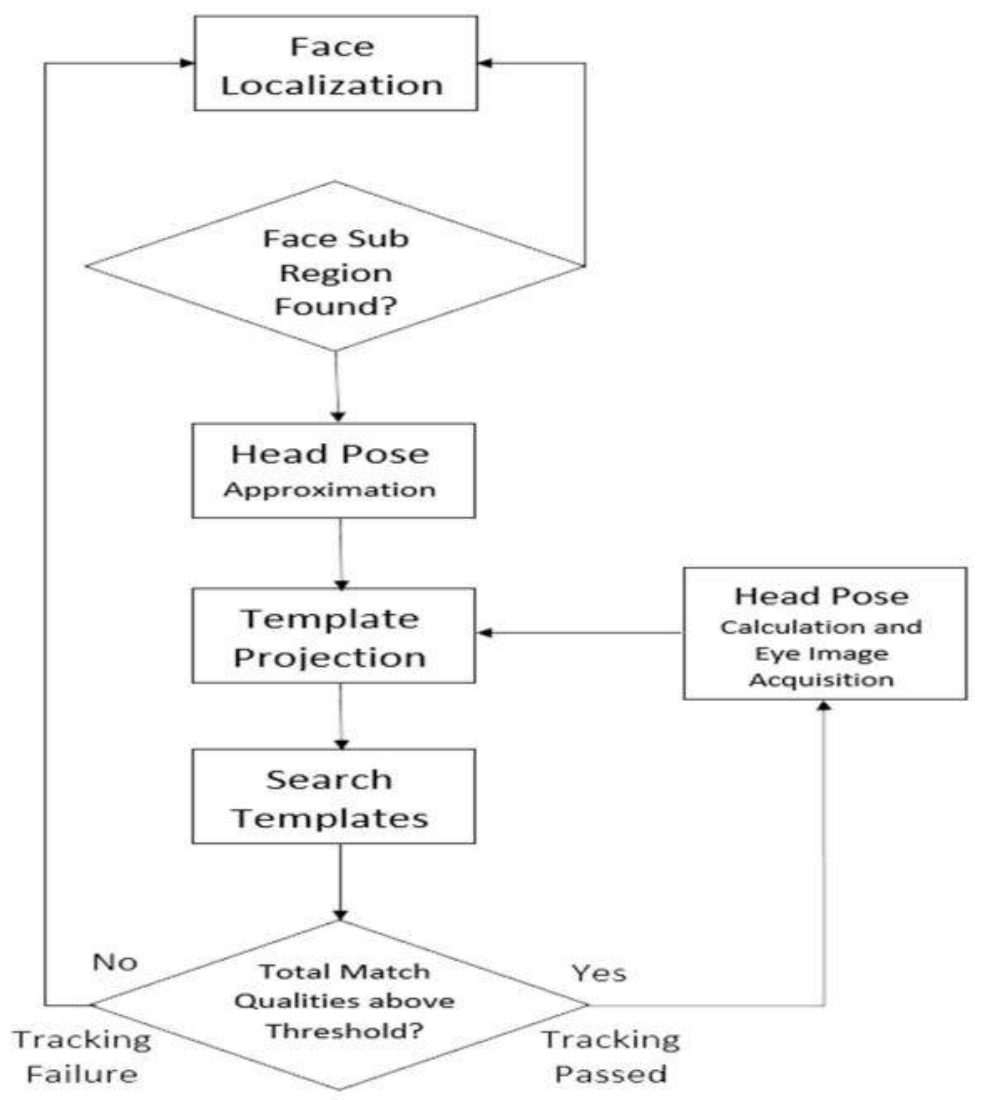

Figure. 3: Head Pose and Eye Image Acquisition

Facial expression detection: Robots convey a social estimation to human PC interaction and tend us to expect in new strategies around that how PC frameworks will be used in step by step life. no holds barred verbal trade is an ongoing way working at once scale in the request of forty milliseconds. the degree of vulnerability right now scale is tremendous, making it important for individuals and machines to depend on tactile well off perceptual natives set up of moderate representative surmising methods. In this paper improvement on one such perceptual crude is examined. The contraption mechanically recognizes frontal faces inside the video circle and codes them with acknowledge to 7 measurements in real time: unprejudiced, outrage, disturb, stress, satisfaction, misery, wonder. The face discoverer utilizes a course of highlight finders talented with boosting strategies. The appearance recognizer gets picture patches situated by utilizing the face finder. A Gabor portrayal of the fix is molded and after that prepared by method for a bank of SVM classifiers. A novel blend of Adaboost and SVM's supplements execution. The gadget was inspected at the Cohn-Kanade dataset of postured outward appearances [7]. The speculation execution to new subjects for a 7-way compelled inclination precise. Greatest curiously the yields of the classifier change effortlessly as a normal for time, giving a without a doubt valuable portrayal to code facial highlights flow in a totally programmed and subtle way. The framework has been sent on broad kind of stages that incorporates Sony's Aibo puppy robot, ATR's RoboVie, and CU illustrator, and is as of now being assessed for bundles comprehensive of automated examining guides, appraisal of human-robot communication. 


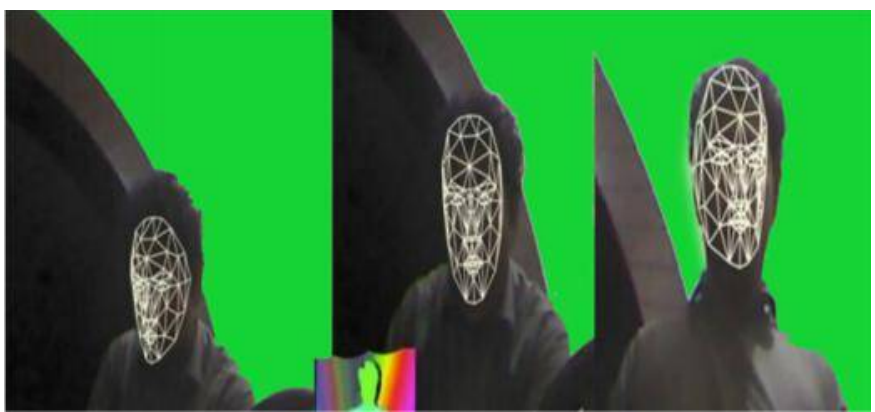

Figure. 4: Facial Structure and expressions Detection

Voice pitch detection (multimodal approach): A proposal was made to interact multimodality between the robot and the person, using voice and gestures, in order to make a friendlier relationship between man and robot. This function has promised to integrate into a service called Humanoid Donaxi, from robotics laboratory of UPAEP. As a result of this research some preliminary results has been achieved so far, this research was the beginning phase.

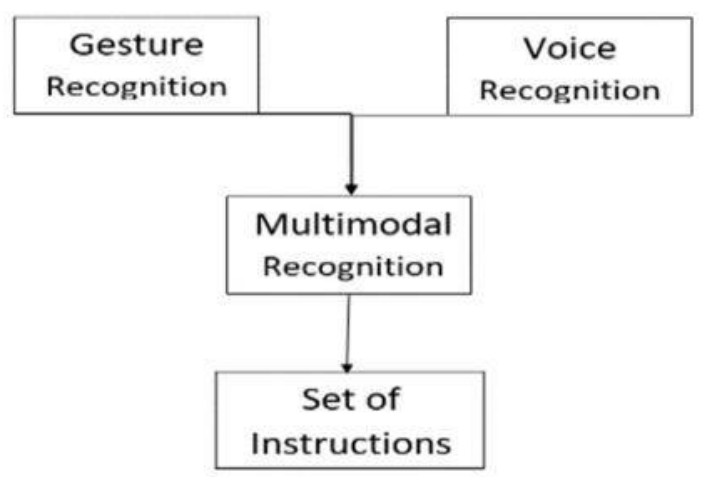

Figure.5: Detection of vocal and gestural response

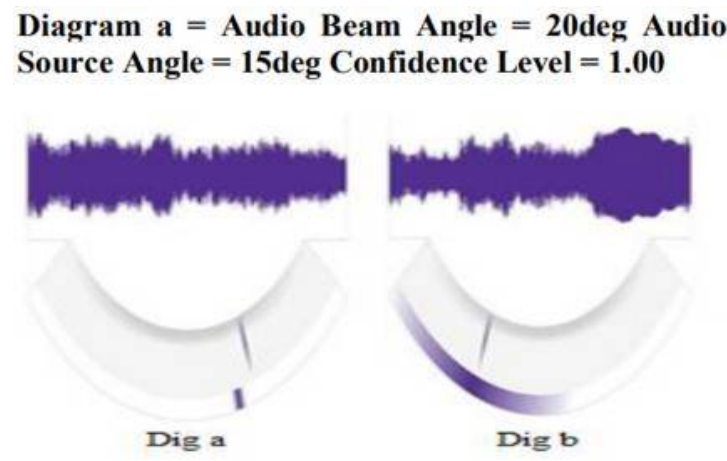

Figure. 6: Voice Pitch Level Variations

Self Balancing: The system architecture comprises a pair of DC motor and an Arduino microcontroller board; a single-axis gyroscope and a 2-axis accelerometer are employed for attitude determination. In addition, a complementary filter is implemented to compensate for gyro drifts. Electrical and kinematic parameters are determined experimentally; PID and LQR- based PI-PD control designs, respectively, are performed on the linearized equations of motion. Experimental results show that self-balancing can be achieved with PI-PD control in the vicinity of the upright position. 


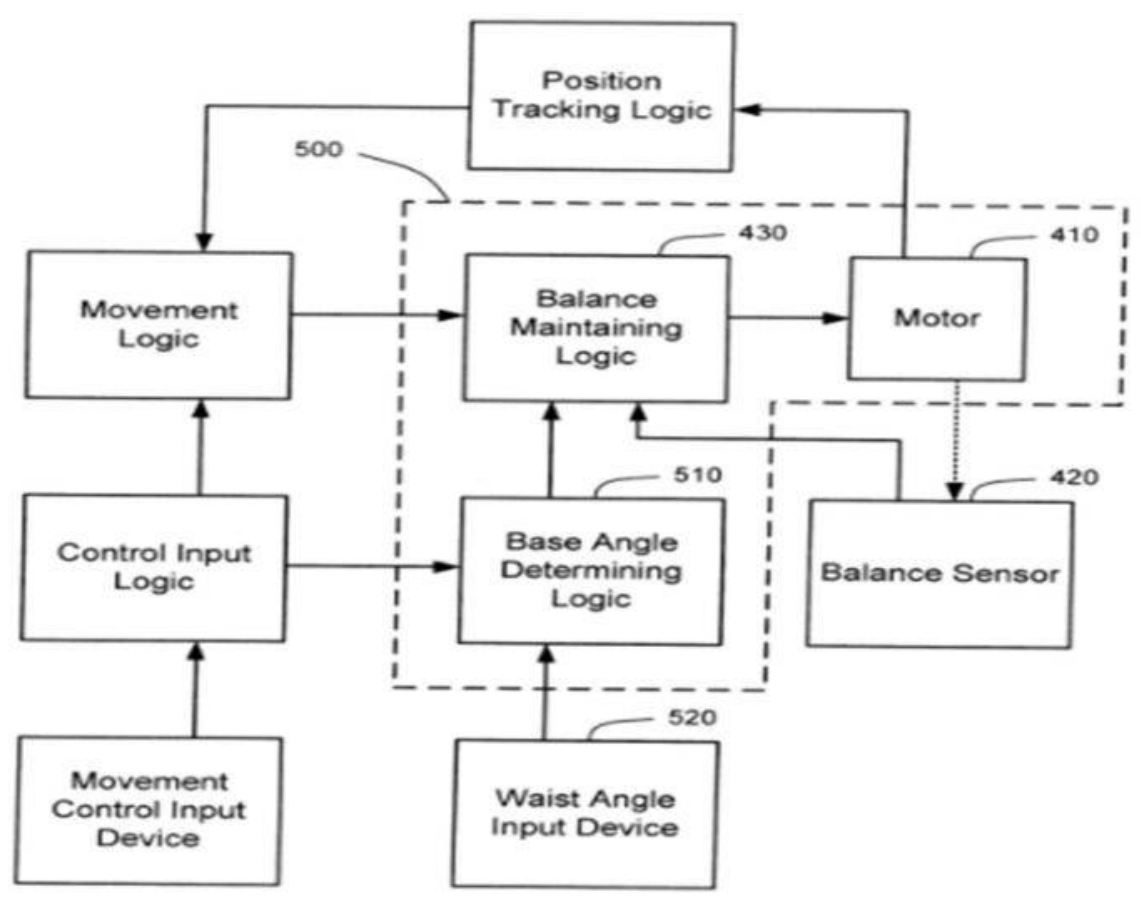

Figure. 7: Flow of Balancing of an agent

3. Proposed work: The Advancement of Robot-Upgraded treatment for youngsters with extreme task will outline robots that can work self-governing and help the specialist to enhance the social cooperation aptitudes, for instance, turn-taking, impersonation and joint consideration. Robot-helped treatments have indicated guarantee as potential evaluation and restorative devices as research has demonstrated that kids with a mental imbalance issue (ASD) connect with more promptly with robots as opposed to people, since robots are straightforward and unsurprising. Be that as it may, current social robots are essentially remote-controlled by the advisors and like standard treatments, still require a ton of time, vitality and HR. The Fantasy Task intends to build up a self-sufficient robot that limits the advisor's mediation so they can concentrate more on the kid and enhance the result of the treatment. The robot will likewise work as a symptomatic apparatus by gathering clinical information amid treatment. The fundamental purpose of this paper is to present such a framework that can be used by robots and they can catch and break down tactile information from the youngsters movement signals, look, outward appearances, sound and voice - and influence the robot to comprehend what the kid is doing as such that they can have a superior association.

The robot is intended to be approachable and interesting for kids with ASD. Key features for it are walk, talk and even model human outward appearances. It never gets baffled or tired. It reliably conveys lessons in a way that kids with ASD react to. This repeating positive experience will make a domain in which kids will learn and flourish. It will enable students to enhance their social and behavioral abilities and pick up the certainty they have to succeed scholastically and socially. This will help people with ASD to figure out how to: Tune in on feelings, Express sympathy, Act in social circumstances, Self-inspire, Sum up in the populace. It is intended to teach social behaviors and emotional identification to kids ages 5-17 who meet the Prerequisite Skills. Expected Services by it are Home Security, Smart Home, Social Interaction, Personal Assistant, Therapist, Multimedia, Edutainment and Elder Care.

Memory Based Approach: Working memory architecture an agent has three types of memory, namely a chronologically ordered belief-set (declarative memory), a set of situated action rules, and a set of production rules (procedural memory). This architecture of an agent consists of a STM and two separate LTMs. 


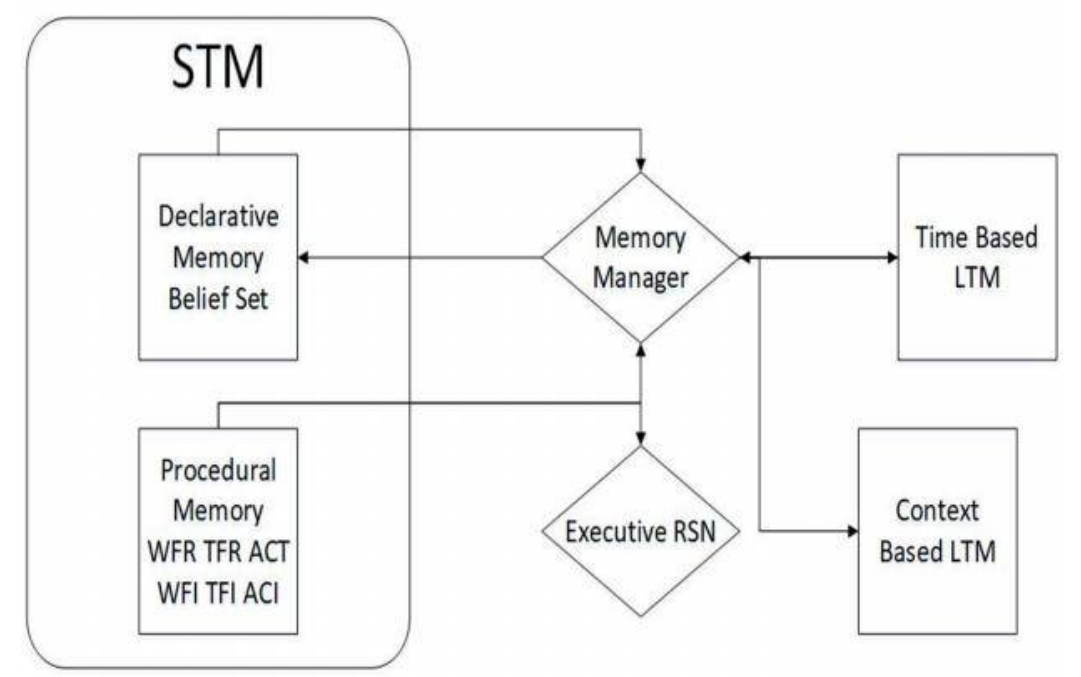

Figure. 8: Memory Based Management

In fig. 8 a flow is demonstrated according to what the robot will store and memorize things about kids and will decided either to keep that for long term purposes or short term.

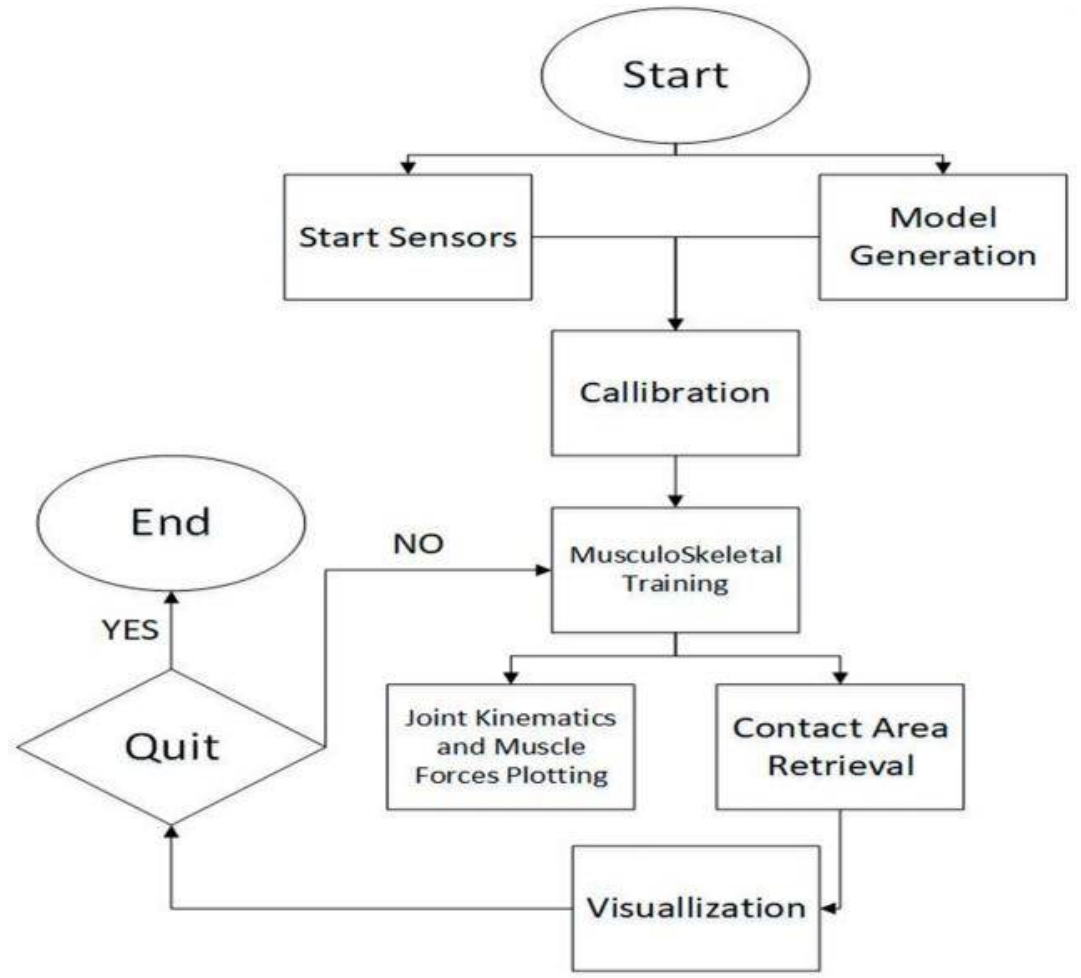

Figure. 9: Body Joints Kinematics flow

In fig. 9 a flow is demonstrated according to what the sensors of robot will execute and start capturing the motion of a kid and start calibrating their structure on the robots predefined feeded biped structure just like a shadpw mode that will allow the robot to understand the movement of kid. 


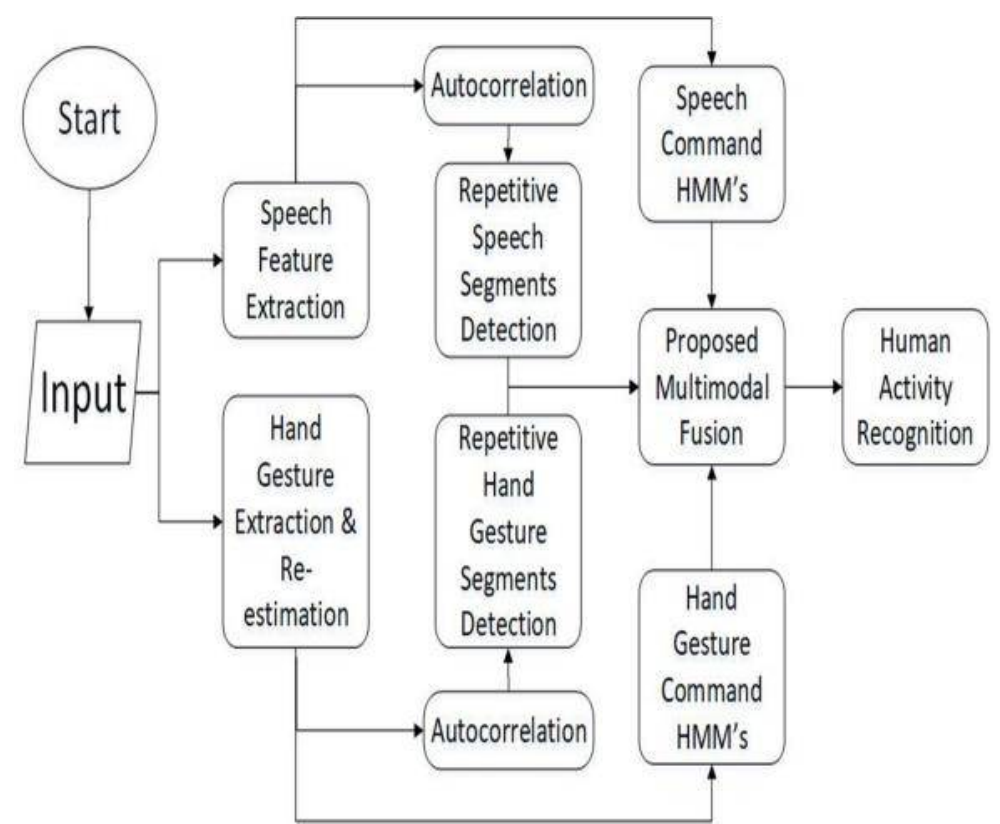

Figure. 10: Speech and Gestural/Postural Detection Flow

In fig. 10 a flow is demonstrated according to what the audio sensors of robot will execute and will calibrate with the motion detection readings so that a data can be created that will make robot understand the kid more and it will try to make a sequence from random obtained values.

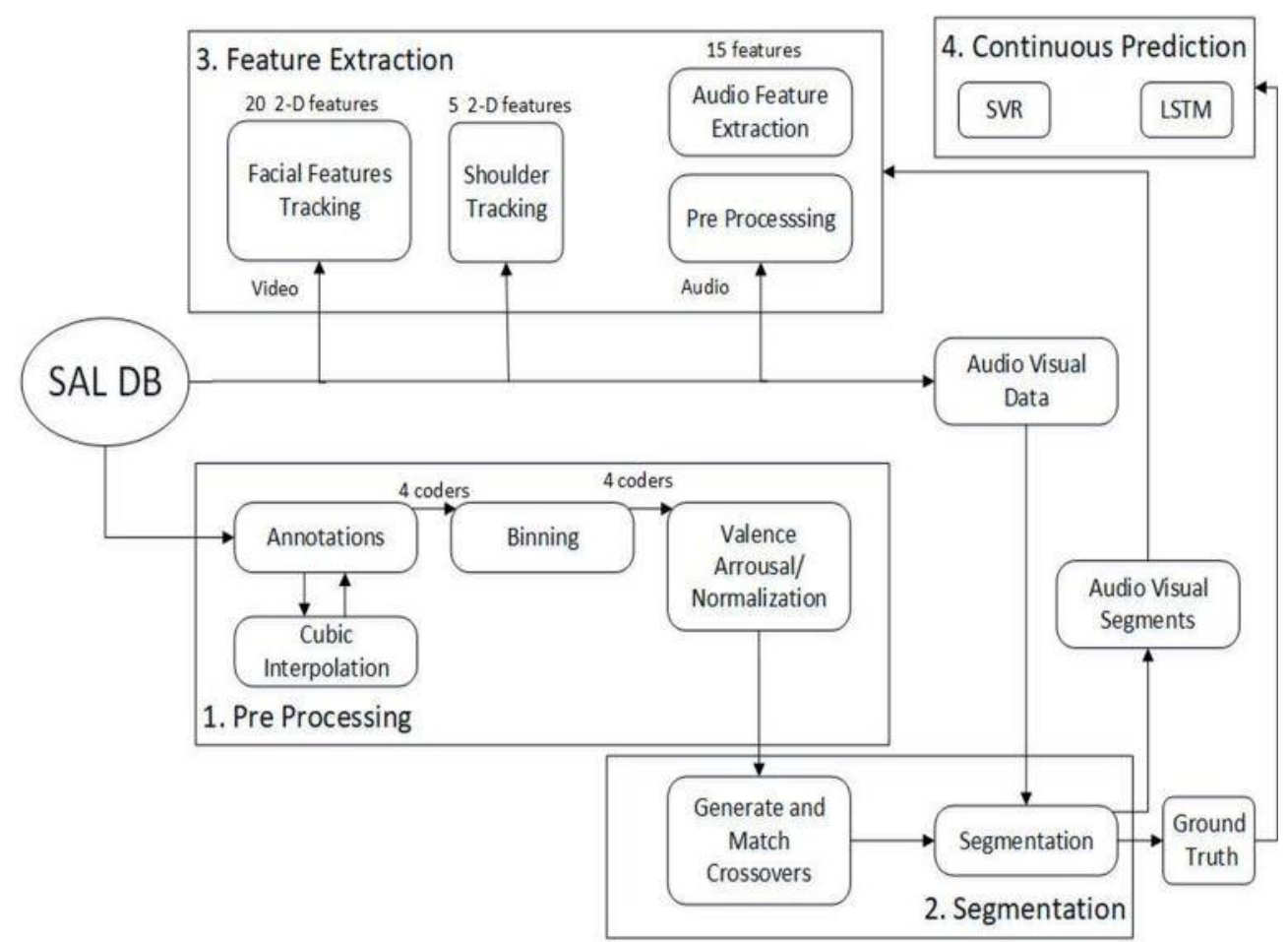

Figure. 11: Overall Flow of Prediction 
In fig. 11 an overall flow is presented where all the extractions and detections are made and then all the data is being sent to predict the possible situation and requirement of a kid.

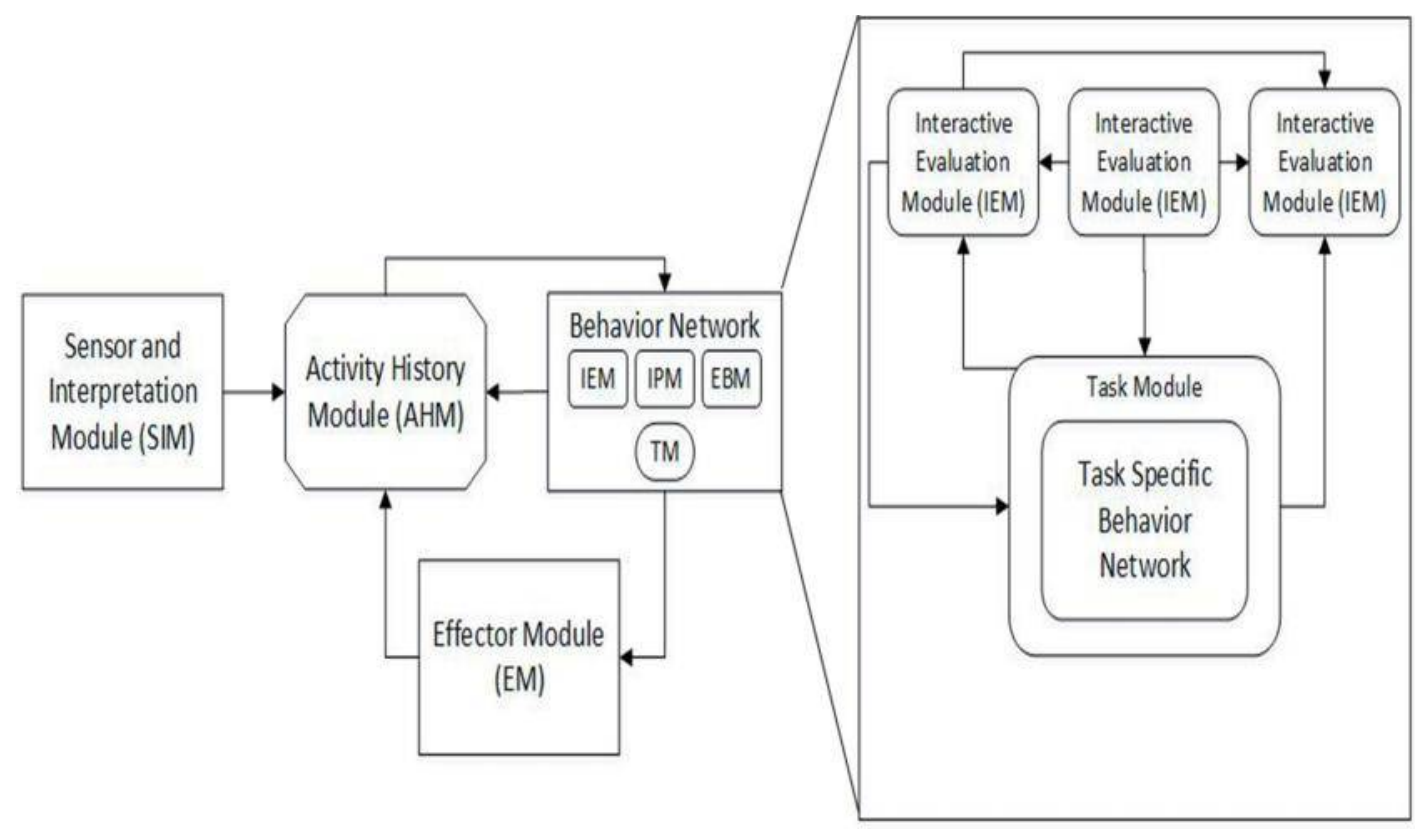

Figure. 12: Behavioral Analysis

In fig. 12 Process for extracting behavioral analysis values is presented. Through this behavior can be categorized for a deep study of a kid's condition.

In all these figures from fig. 1 to fig. 12 the structure is being presented that how a robot can be aware of a child with ASD's condition. There will be need of a camera and Infrared sensor that can detect the body/biped movement of a child and can notice or take readings of movement later to compare with other aspects, then the ultrasonic/ distance sensor will be needed to measure the distance and make the body movement readings more accurate on different situations and timing, 4 mics will be needed to clearly capture the voice and clearly understand what a child will say as a child with ASD condition speaks fast and in a low pitch voice. Also, another infrared and camera will be planted to detect the facial expressions and features, and a eye gaze camera to detect the random movement of eyes. After all these values will be extracted. These will get compared according to the current situation and will be stored in the database for therapists to deeply study them and robot will not only store them but also will predict on the basis of predefined possible datasets given by therapists stored in cloud. Now the predefined action/ need will be get done if that will satisfy the kid then the most probable action will be predefined but if the condition/ hyper nature of that kid will remain same then the robot will perform some basic actions in order to calm the kid and the action that will result in positive will be marked as new most probable action. For emergency cases as the kids with ASD are not self-aware and can get in any emergency situation so being a friendly robot. There will be need of some emergency protocols that will include heart beat sensors and call for doctor or guardian in case of some movement other than usual. A LCD will be placed on that robot for entertainment and learning purposes of the robot to give them lessons regularly according to the behavioral learning. If repetition and insisted things will seem to work then the according protocol will execute to help the kid in learning. 
4. Conclusion and future work: It is a venture that will convey the cutting edge treatment, socially dynamic benevolent, security convention based and assistive robots, and its center is its psychological model which deciphers tactile information (body development and feeling appearance signals), utilizes these observations to evaluate the kid's conduct by figuring out how to delineate to advisor particular behavioral classes, and afterward figures out how to outline kid practices to fitting robot activities as determined by the specialists. The multi-tangible information that we are catching will be utilized to give quantitative help to the conclusion and care and treatment of ASD, supplanting current work escalated systems including paper and pencil, or manual video examination. It is implementable and can help the field of treatments heavily. Future of treatments rely on the learning and experience of robots that will be more precise and fast than the humanly examination. It is a step forward to achieve that using cloud computing and sharing of personal experience. Next step is to implement it using the theoretical framework presented in this paper.

\section{REFERENCES}

[1] Duquette, A., Michaud, F., \& Mercier, H. (2008). Exploring the use of a mobile robot as an imitation agent with children with low-functioning autism. Autonomous Robots, 24(2), 147-157.

[2] Robins, B., Dickerson, P., Stribling, P., \& Dautenhahn, K. (2004). Robot-mediated joint attention in children with autism: A case study in robot-human interaction. Interaction studies, 5(2), 161-198.

[3] Stanton, C. M., Kahn Jr, P. H., Severson, R. L., Ruckert, J. H., \& Gill, B. T. (2008, March). Robotic animals might aid in the social development of children with autism. In Proceedings of the $3 \mathrm{rd}$ ACM/IEEE international conference on Human robot interaction (pp. 271-278). ACM.

[4] Tanaka, F., Cicourel, A., \& Movellan, J. R. (2007). Socialization between toddlers and robots at an early childhood education center. Proceedings of the National Academy of Sciences, 104(46), 17954-17958.

[5] Feil-Seifer, D., \& Mataric, M. J. (2005, June). Defining socially assistive robotics. In Rehabilitation Robotics, 2005. ICORR 2005. 9th International Conference on (pp. 465-468). IEEE.

[6] Zaraki, A., Mazzei, D., Giuliani, M., \& De Rossi, D. (2014). Designing and evaluating a social gaze-control system for a humanoid robot. IEEE Transactions on Human-Machine Systems, 44(2), 157-168.

[7] Prodanov, P. J., Drygajlo, A., Ramel, G., Meisser, M., \& Siegwart, R. (2002). Voice enabled interface for interactive tour-guide robots. In Intelligent Robots and Systems, 2002. IEEE/RSJ International Conference on (Vol. 2, pp. 1332-1337). IEEE.

[8] Rosenholtz, R., Huang, J., \& Ehinger, K. A. (2012). Rethinking the role of top-down attention in vision: Effects attributable to a lossy representation in peripheral vision. Frontiers in psychology, 3, 13.

[9] Yoshikawa, Y., Shinozawa, K., Ishiguro, H., Hagita, N., \& Miyamoto, T. (2006, October). The effects of responsive eye movement and blinking behavior in a communication robot. In Intelligent Robots and Systems, 2006 IEEE/RSJ International Conference on (pp. 4564-4569). IEEE.

[10] Takahashi, T., Nakanishi, S., Kuno, Y., \& Shirai, Y. (1998, October). Human-robot interface by verbal and nonverbal behaviors. In Intelligent Robots and Systems, 1998. Proceedings., 1998 IEEE/RSJ International Conference on (Vol. 2, pp. 924-929). IEEE.

[11] Nam, H., Kim, H. S., Kwon, Y., \& Yang, S. I. (1998, October). Speaker verification system using hybrid model with pitch detection by wavelets. In Time-Frequency and Time-Scale Analysis, 1998. Proceedings of the IEEE-SP International Symposium on (pp. 153-156). IEEE.

[12] Kadambe, S., \& Boudreaux-Bartels, G. F. (1992). Application of the wavelet transform for pitch detection of speech signals. IEEE transactions on Information Theory, 38(2), 917-924.

[13] Jing, L. I., \& Changchun, B. A. O. (2002, August). A pitch detector based on the dyadic wavelet transform and the autocorrelation function. In Signal Processing, 2002 6th International Conference on (Vol. 1, pp. 414-417). IEEE.

[14] Obaidat, M. S., Lee, T., Zhang, E., Khalid, G., \& Nelson, D. (1996, October). Wavelet algorithm for the estimation of pitch period of speech signal. In Electronics, Circuits, and Systems, 1996. ICECS'96., Proceedings of the Third IEEE International Conference on (Vol. 1, pp. 471-474). IEEE.

[15] Kader, N. A. (2000). Pitch detection algorithm using a wavelet correlation model. In Radio Science Conference, 2000. 17th NRSC'2000. Seventeenth National (pp. C33-1). IEEE. 
[16] Sierhuis, M., \& Hoof, R. (2004). On Short-Term and Long-Term Memory for Brahms Agents. In AAAI Spring Symposium 2004 Workshop on Interaction between Humans and Autonomous Systems over Extended Operations (pp. 29-34).

[17] Thrun, S., Bennewitz, M., Burgard, W., Cremers, A. B., Dellaert, F., Fox, D., ... \& Schulz, D. (1999). MINERVA: A second-generation museum tour-guide robot. In Robotics and automation, 1999. Proceedings. 1999 IEEE international conference on (Vol. 3). IEEE.

[18] Vasquez, H., Vargas, H. S., \& Sucar, L. E. (2015). Using Gestures to Interact with a Service Robot using Kinect 2. Research in Computing Science, 96, 85-93.

[19] Zhijin, X., \& Jie, W. (2000). A new pitch detection method. In Signal Processing Proceedings, 2000. WCCC-ICSP 2000. 5th International Conference on (Vol. 2, pp. 747-751). IEEE. 